\title{
A VECTOR-VALUED SHARP MAXIMAL INEQUALITY ON MORREY SPACES WITH NON-DOUBLING MEASURES
}

\author{
YOSHIHIRO SAWANO
}

\begin{abstract}
We consider the vector-valued extension of the Fefferman-SteinStrömberg sharp maximal inequality under growth condition. As an application we obtain a vector-valued extension of the boundedness of the commutator. Furthermore, we prove the boundedness of the commutator.
\end{abstract}

2000 Mathematics Subject Classification: Primary 42B35; Secondary $42 \mathrm{~B} 25$.

Key words and phrases: Weighted norm inequality, non-doubling (nonhomogeneous), sharp-maximal function, commutator.

\section{INTRODUCTION}

In this paper we obtain a vector-valued extension of the sharp-maximal inequality and develop its applications.

We denote by $M$ the Hardy-Littlewood maximal operator and by $M^{\sharp}$ as the sharp maximal operator. We mean the sharp maximal inequality by one of the form:

$$
\left\|M f: L^{p}\left(\mathbf{R}^{d}\right)\right\| \leq C\left\|M^{\sharp} f: L^{p}\left(\mathbf{R}^{d}\right)\right\| \quad(1<p<\infty),
$$

which appeared in [3]. The primary aim of this paper is to obtain an inequality of the form

$$
\left\|\left(\sum_{j=1}^{\infty} M f_{j}^{q}\right)^{\frac{1}{q}}: L^{p}(\mu)\right\| \leq C\left\|\left(\sum_{j=1}^{\infty} M^{\sharp} f_{j}^{q}\right)^{\frac{1}{q}}: L^{p}(\mu)\right\| \quad(1<p, q<\infty) .
$$

Here and below $C$ will be used for constants that may change from one occurrence to another. Constants with subscripts, such as $C_{0}, C_{1}, C_{2}$, do not change in different occurrences. Throughout this paper $\mu$ will be a (positive) Radon measure on $\mathbf{R}^{d}$ satisfying the growth condition:

$$
\mu(B(x, l)) \leq C_{0} l^{n} \text { for all } x \in \operatorname{supp}(\mu) \text { and } l>0,
$$

where $n, 0<n \leq d$, is a fixed number and $B(x, r)$ is a ball with center $x$ and ra$\operatorname{dius} r>0$. We do not assume the doubling condition $\mu(B(x, 2 r)) \leq C \mu(B(x, r))$ $(x \in \operatorname{supp}(\mu), r>0)$. We are going to obtain (1) with the underlying measure $\mu$ satisfying only the growth condition and the function space will be the Morrey space, which is an extension of the $L^{p}$ space.

By a "cube" $Q \subset \mathbf{R}^{d}$ we mean a compact cube whose edges are parallel to the coordinate axes. Its side length will be denoted by $\ell(Q)$. For $c>0, c Q$ will denote a cube concentric to $Q$ with its sidelength $c \ell(Q)$. The set of all 
cubes $Q \subset \mathbf{R}^{d}$ with positive $\mu$-measure will be denoted by $\mathcal{Q}(\mu)$. We recall the definition of Morrey spaces with non-doubling measures.

Let $k>1$ and $1 \leq q \leq p<\infty$. We define a Morrey space $\mathcal{M}_{q}^{p}(k, \mu)$ as

$$
\mathcal{M}_{q}^{p}(k, \mu):=\left\{f \in L_{l o c}^{q}(\mu):\left\|f \mid \mathcal{M}_{q}^{p}(k, \mu)\right\|<\infty\right\},
$$

where the norm $\left\|f \mid \mathcal{M}_{q}^{p}(k, \mu)\right\|$ is given by

$$
\left\|f \mid \mathcal{M}_{q}^{p}(k, \mu)\right\|:=\sup _{Q \in \mathcal{Q}(\mu)} \mu(k Q)^{\frac{1}{p}-\frac{1}{q}}\left(\int_{Q}|f(x)|^{q} d \mu(x)\right)^{\frac{1}{q}} .
$$

By applying Hölder's inequality to (3), it is easy to see that

$$
L^{p}(\mu)=\mathcal{M}_{p}^{p}(k, \mu) \subset \mathcal{M}_{q_{2}}^{p}(k, \mu) \subset \mathcal{M}_{q_{1}}^{p}(k, \mu)
$$

for $1 \leq q_{1} \leq q_{2} \leq p<\infty$. The definition of Morrey spaces does not depend on a constant $k>1$. The norms for different choices of $k>1$ are equivalent. For details we refer to [13]. Nevertheless, for definiteness, we will assume $k=2$ in the definition and denote $\mathcal{M}_{q}^{p}(2, \mu)$ by $\mathcal{M}_{q}^{p}(\mu)$.

Our BMO here is a RBMO (regular bounded mean oscillation) introduced by $\mathrm{X}$. Tolsa which is a suitable substitute for the classical spaces [16]. We adopt the notation due to Chen and Sawyer, who modified the notion of Tolsa in order to develop the theory of commutators of a fractional integral operator.

\section{Definition 1.1.}

(1) Let $0 \leq \alpha<n$. Given two cubes $Q \subset R \in \mathcal{Q}(\mu)$, we set

$$
K_{Q, R}^{(\alpha)}:=1+\sum_{k=0}^{N_{Q, R}}\left(\frac{\mu\left(2^{k} Q\right)}{\ell\left(2^{k} Q\right)^{n}}\right)^{1-\alpha / n}
$$

where $N_{Q, R}$ is the smallest integer $k \geq 0$ such that $2^{k} Q \supset R$. For simplicity, we denote $K_{Q, R}=K_{Q, R}^{(0)}$.

(2) We say that $Q \in \mathcal{Q}(\mu)$ is a doubling cube if $\mu(2 Q) \leq 2^{d+1} \mu(Q)$. We denote by $\mathcal{Q}(\mu, 2)$ the set of all doubling cubes.

(3) Given $Q \in \mathcal{Q}(\mu)$, we set $Q^{*}$ as the smallest doubling cube $R$ of the form $R=2^{j} Q$ with $j \in \mathbf{N}_{0}:=\{0\} \cup \mathbf{N}$.

(4) We say that $f \in L_{l o c}^{1}(\mu)$ is an element of RBMO if it satisfies

$$
\sup _{Q \in \mathcal{Q}(\mu)} \frac{1}{\mu\left(\frac{3}{2} Q\right)} \int_{Q}\left|f(y)-m_{Q^{*}}(f)\right| d \mu(y)+\sup _{\substack{Q \subset R \\ Q, R \in \mathcal{Q}(\mu, 2)}} \frac{\left|m_{Q}(f)-m_{R}(f)\right|}{K_{Q, R}}<\infty
$$

where $m_{Q}(f):=\frac{1}{\mu(Q)} \int_{Q} f(x) d \mu(x)$. We denote this quantity by $\|f\|_{*}$.

By the growth condition (2) there are a lot of big doubling cubes. Speaking precisely, given any cube $Q \in \mathcal{Q}(\mu)$, we can find $j \in \mathbf{N}$ with $2^{j} Q \in \mathcal{Q}(\mu, 2)$. Meanwhile, for $\mu$-a.e. $x \in \mathbf{R}^{d}$, there exists a sequence of doubling cubes $\left\{Q_{k}\right\}_{k}$ 
centered at $x$ with $\ell\left(Q_{k}\right) \rightarrow 0$ as $k \rightarrow \infty$. So we can say that there are a lot of small doubling cubes, too. (See [16].)

For $f \in L_{l o c}^{1}(\mu)$ we define two maximal operators mainly due to Tolsa (see [16]): Let $0 \leq \alpha<n$. The sharp maximal operator $M^{\sharp, \alpha} f(x)$ is defined as

$$
\begin{aligned}
M^{\sharp, \alpha} f(x):= & \sup _{x \in Q \in \mathcal{Q}(\mu)} \frac{1}{\mu\left(\frac{3}{2} Q\right)} \int_{Q}\left|f(y)-m_{Q^{*}}(f)\right| d \mu(y) \\
& +\sup _{\substack{x \in Q \subset R \\
Q, R \in \mathcal{Q}(\mu, 2)}} \frac{\left|m_{Q}(f)-m_{R}(f)\right|}{K_{Q, R}^{(\alpha)}}
\end{aligned}
$$

and $N f(x)$ is defined as $N f(x):=\sup _{x \in Q \in \mathcal{Q}(\mu, 2)} m_{Q}(|f|)$. The modification parameter $\alpha$ was introduced by Chen and Sawyer. We also introduce a $\kappa$-times maximal operator

$$
M_{\kappa} f(x):=\sup _{x \in Q \in \mathcal{Q}(\mu)} \frac{1}{\mu(\kappa Q)} \int_{Q}|f(x)| d \mu(x) \quad(\kappa>1) .
$$

Since there are a lot of doubling cubes, we have a pointwise estimate

$$
|f(x)| \leq N f(x),|f(x)| \leq \kappa^{d+1} M_{\kappa} f(x)
$$

for $\mu$-almost all $x \in \mathbf{R}^{d}$. It is known that $M_{\kappa}: \mathcal{M}_{q}^{p}(\mu) \rightarrow \mathcal{M}_{q}^{p}(\mu)$ is a bounded operator (cf. [13]), if $\kappa>1$. If $\mu$ is a finite measure, we denote

$$
m_{\mathbf{R}^{d}}(f):=\frac{1}{\mu\left(\mathbf{R}^{d}\right)} \int_{\mathbf{R}^{d}} f(x) d \mu(x) .
$$

Proposition $1.2([14])$. Suppose that $1<q \leq p<\infty, 0 \leq \alpha<n$.

(1) For any $f \in L_{\text {loc }}^{1}(\mu)$, there exists a constant $C>0$ independent of $f$ such that

$$
\left\|N f: \mathcal{M}_{q}^{p}(\mu)\right\| \leq C\left(\left\|M^{\sharp, \alpha} f: \mathcal{M}_{q}^{p}(\mu)\right\|+\left\|f: \mathcal{M}_{1}^{p}(\mu)\right\|\right) .
$$

(2) Suppose that there exists an increasing sequence of concentric doubling cubes

$$
I_{0} \subset I_{1} \subset \cdots \subset I_{k} \subset \cdots
$$

such that

$$
\lim _{k \rightarrow \infty} m_{I_{k}}(f)=0 \text { and } \bigcup_{k} I_{k}=\mathbf{R}^{d}
$$

Then there exists a constant $C>0$ independent of $f$ such that

$$
\left\|N f: \mathcal{M}_{q}^{p}(\mu)\right\| \leq C\left\|M^{\sharp, \alpha} f: \mathcal{M}_{q}^{p}(\mu)\right\| .
$$

In particular, if $\mu$ is finite, (6) is available for all $f$ with $m_{\mathbf{R}^{d}}(f)=0$.

(3) Suppose that $\mu\left(\mathbf{R}^{d}\right)<\infty$. Then we have

$$
\left\|N f: \mathcal{M}_{q}^{p}(\mu)\right\| \leq C\left(\left\|M^{\sharp, \alpha} f: \mathcal{M}_{q}^{p}(\mu)\right\|+\left\|f: L^{1}(\mu)\right\|\right) .
$$


In this paper we prove the vector-valued extension of (2) and (3) of the previous proposition. Since $\mathcal{M}_{1}^{p}(\mu)$ contains $\mathcal{M}_{q}^{p}(\mu)$, the norms in (1) are equivalent. Condition (5) will be a key to our argument. Below we give our main results.

Theorem 1.3. Suppose that $1 \leq q \leq p<\infty, 1<r<\infty, \kappa>1$ and $0 \leq \alpha<n$. Let $f_{j} \in \mathcal{M}_{q}^{p}(\mu)$ with $j=1,2, \ldots$.

(1) Assume that $\mu\left(\mathbf{R}^{d}\right)=\infty$. Then we have

$$
\left\|\left(\sum_{j=1}^{\infty} N f_{j}{ }^{r}\right)^{\frac{1}{r}}: \mathcal{M}_{q}^{p}(\mu)\right\| \leq\left\|\left(\sum_{j=1}^{\infty} M^{\sharp, \alpha} f_{j}{ }^{r}\right)^{\frac{1}{r}}: \mathcal{M}_{q}^{p}(\mu)\right\| .
$$

(2-a) Assume that $\mu\left(\mathbf{R}^{d}\right)<\infty$. If $m_{\mathbf{R}^{d}}\left(f_{j}\right)=0$ for all $j=1,2, \ldots$, then we have (7).

(2-b) Assume that $\mu\left(\mathbf{R}^{d}\right)<\infty$. Then we have for all $\left\{f_{j}\right\}_{j=1}^{\infty} \subset \mathcal{M}_{q}^{p}(\mu)$

$$
\begin{aligned}
& \left\|\left(\sum_{j=1}^{\infty} N f_{j}^{r}\right)^{\frac{1}{r}}: \mathcal{M}_{q}^{p}(\mu)\right\| \\
& \leq C\left\|\left(\sum_{j=1}^{\infty} M^{\sharp, \alpha} f_{j}^{r}\right)^{\frac{1}{r}}: \mathcal{M}_{q}^{p}(\mu)\right\|+C\left\{\sum_{j=1}^{\infty}\left(\int_{\mathbf{R}^{d}}\left|f_{j}(x)\right| d \mu(x)\right)^{r}\right\}^{\frac{1}{r}} .
\end{aligned}
$$

At first glance the condition $f_{j} \in \mathcal{M}_{q}^{p}(\mu)$ seems to be superfluous, but this assumption can be verified by using Proposition 1.2. So this seemingly strong assumption suffices. It is easy to see that $M^{\sharp, \alpha}$ is bounded pointwise by $M_{2}$. Since we have the Fefferman-Stein type inequality for $M_{2}$ on $L^{p}(\mu)$ spaces and on Morrey spaces $\mathcal{M}_{q}^{p}(\mu)$ (see [12] and [13]), it follows that the right-hand and the left-hand sides of the formulae of Theorem 1.3 are equivalent. By using Minkowski's inequality and $\mu\left(\mathbf{R}^{d}\right)<\infty$ the equivalence in (2-b) also holds. Theorem 1.3 (2-b) can be easily obtained from Theorem 1.3 (2-a). Indeed, given a system of functions $f_{j} \in \mathcal{M}_{q}^{p}(\mu)(j=0,1, \ldots)$, we have that $f_{j}-m_{\mathbf{R}^{d}}\left(f_{j}\right)$ $(j=0,1, \ldots)$ satisfies the assumption of Theorem $1.3(2-\mathrm{a})$. Thus we have

$$
\left\|\left(\sum_{j=1}^{\infty}\left(N\left(f_{j}-m_{\mathbf{R}^{d}}\left(f_{j}\right)\right)\right)^{r}\right)^{\frac{1}{r}}: \mathcal{M}_{q}^{p}(\mu)\right\| \leq C\left\|\left(\sum_{j=1}^{\infty} M^{\sharp, \alpha} f_{j}{ }^{r}\right)^{\frac{1}{r}}: \mathcal{M}_{q}^{p}(\mu)\right\|,
$$

which yields Theorem $1.3(2-\mathrm{b})$. Now it follows that we have only to prove Theorem 1.3 (1) and (2-a). In both cases we have (5).

Remark 1.4. It is worth restating Theorem 1.3 in the case of a Lebesgue space $L^{p}(d x)$. Notice that if $\mu=d x$, then $M^{\sharp, 0} f(x)$ is equivalent to the usual sharp maximal function introduced and studied in [3]. Applying our result with $\mu=$ $d x$ and $1<p=q<\infty$ and using the Fefferman-Stein vector-valued inequality, we have a norm equivalence for any countable subset $\left\{f_{j}\right\}_{j=1}^{\infty} \subset L^{p}(d x)$

$$
\left\|\left(\sum_{j=1}^{\infty} M f_{j}^{r}\right)^{\frac{1}{r}}: L^{p}(d x)\right\| \sim\left\|\left(\sum_{j=1}^{\infty} M^{\sharp, \alpha} f_{j}^{r}\right)^{\frac{1}{r}}: L^{p}(d x)\right\| .
$$


As an application of Theorem 1.3 we obtain the vector-valued extension of the boundedness of commutators. Under a commutator we mean an operator of the form $[a, T] f(x)=a(x) T f(x)-T(a f)(x)$, where $a$ is a function and $T$ is a bounded operator. The classical results say that $[a, T]$ is a bounded operator from $L^{p}(d x)$ to $L^{p}(d x)$ if $a \in \mathrm{BMO}$ and $T$ is a Calderón-Zygmund operator and that $[a, T]$ is a bounded operator from $L^{p}(d x)$ to $L^{q}(d x)$ if $a \in \mathrm{BMO}$ and $T$ is a fractional integral operator, where $p$ and $q$ are suitable real numbers. Fazio and Ragusa [11] extended these results to the classical Morrey spaces. The definition will be given in the next section.

\section{Preliminaries}

In this section we collect the known facts on maximal operators, weighted norm inequalities and commutator operators.

In what follows we will use the notation due to Triebel [17] to state a vectorvalued inequality. Let $X$ be $\mathcal{M}_{q}^{p}(\mu)$ or $L^{p}(\mu)$ with $1 \leq q \leq p<\infty$ and let $\|\cdot: X\|$ be its norm. We denote

$$
\left\|f_{j}: X\left(l^{r}\right)\right\|:=\left\|\left(\sum_{j=1}^{\infty}\left|f_{j}(\cdot)\right|^{r}\right)^{\frac{1}{r}}: X\right\| .
$$

Thus we are going to prove that

$$
\left\|M_{\kappa} f_{j}: X\left(l^{r}\right)\right\| \leq C\left\|M^{\sharp, \alpha} f_{j}: X\left(l^{r}\right)\right\| .
$$

2.1. Maximal operators. For $f \in L_{l o c}^{1}(\mu), \kappa>1$ and $0 \leq \alpha<n$, a fractional maximal operator $M_{\kappa}^{\alpha} f(x)$ is defined as

$$
M_{\kappa}^{\alpha} f(x):=\sup _{x \in Q \in \mathcal{Q}(\mu)} \frac{1}{\mu(\kappa Q)^{1-\frac{\alpha}{n}}} \int_{Q}|f(x)| d \mu(x) .
$$

It follows that, by definition, $M_{\kappa}^{0}=M_{\kappa}$. As for the boundedness of $M_{\kappa}^{\alpha}$ on the Morrey spaces, a vector-valued inequality of Fefferman-Stein type is known.

Lemma 2.1 ([13]). Suppose that $\kappa>1,0 \leq \alpha<n, 1<q \leq p<\infty$, $1<t \leq s<\infty, 1<r<\infty, 1 / s=1 / p-\alpha / n$ and $t / s=q / p$. Then we have

$$
\left\|M_{\kappa}^{\alpha} f_{j}: \mathcal{M}_{t}^{s}\left(l^{r}\right)\right\| \leq C\left\|f_{j}: \mathcal{M}_{q}^{p}\left(l^{r}\right)\right\|
$$

In particular we have (taking into account that $N f(x) \leq C M_{2} f(x) \mu$-a.e.)

$$
\left\|N f_{j}: \mathcal{M}_{t}^{s}\left(l^{r}\right)\right\| \leq C\left\|f_{j}: \mathcal{M}_{q}^{p}\left(l^{r}\right)\right\|
$$

We use a covering lemma of Besicovitch type.

Lemma 2.2. Let $\kappa>1$. Suppose that $\left\{R_{j}\right\}_{j \in J}$ is a family of cubes with bounded diameters. Then we can find a subset $J_{0}$ in $J$ such that

$$
\bigcup_{j \in J} R_{j} \subset \bigcup_{j \in J_{0}} \kappa R_{j}, \sum_{j \in J_{0}} \chi_{R_{j}}(x) \leq C_{\kappa}
$$

where $\chi_{A}$ is the indicator function of $A \subset \mathbf{R}^{d}$. 
As for a weak-type assertion, we have the following proposition which will be obtained easily by using Lemma 2.2 .

Lemma 2.3. Suppose that $\kappa>1$. Then we have

$$
\mu\left\{x \in \mathbf{R}^{d}: M_{\kappa} f(x)>\lambda\right\} \leq \frac{C_{\kappa}}{\lambda} \int_{\mathbf{R}^{d}}|f(x)| d \mu(x),
$$

where $C_{\kappa}$ is the same constant as in Lemma 2.2 .

2.2. Weighted norm inequality. To prove Theorem 1.3 we use the technique of a weighted norm inequality. In [8], Komori considered weighted norm inequalities with respect to $M_{\kappa}$. He considered weights with Radon measure $\mu$, where $\mu$ does not necessarily satisfy either the doubling condition or the growth condition. In this paper we deal with a class of weights which are "almost" in $A_{1}(\mu)$ and consider the double-weighted norm inequality

$$
\int_{\left\{x \in \mathbf{R}^{d} ; M_{\kappa} f(x)>\lambda\right\}} u(x) d \mu(x) \leq \frac{C}{\lambda} \int_{\mathbf{R}^{d}}|f(x)| v(x) d \mu(x) .
$$

Our problem is that for given $\kappa>1$ we have to find a pair $(u, v)$ with (12). If the measure $\mu$ is doubling, as in [5], this is equivalent to $M_{\kappa} u(x) \leq C v(x)$. The following result is due to Komori [8]. His result contains the $L^{{ }^{p}}$-version but here we need the $L^{1}$-assertion only.

Proposition 2.4. Let $\kappa>\kappa^{\prime}>1$. Suppose that a locally $\mu$-integrable function $w$ satisfies $M_{\kappa^{\prime}} w(x) \leq C w(x)$ for some positive constant $C$. Then the following inequality is true:

$$
\int_{\left\{x \in \mathbf{R}^{d}: M_{\kappa} f(x)>\lambda\right\}} w(x) d \mu(x) \leq \frac{C}{\lambda} \int_{\mathbf{R}^{d}}|f(x)| w(x) d \mu(x) .
$$

Although Komori considered single-weighted norm inequalities, the results can be readily transformed into a double-weighted norm inequality. With a minor modification of the proof of Proposition 2.4, we can prove the following proposition which will be used later.

Proposition 2.5. Let $\kappa>\kappa^{\prime}>1$ and $u, v$ be $\mu$-locally integrable functions. Suppose that $M_{\kappa^{\prime}} u(x) \leq C v(x)$. Then we have

$$
\int_{\left\{x \in \mathbf{R}^{n}: M_{\kappa} f(x)>\lambda\right\}} u(x) d \mu(x) \leq \frac{C}{\lambda} \int_{\mathbf{R}^{n}}|f(x)| v(x) d \mu(x) .
$$

Komori showed that in his class of weights, there is no equivalence as in the classical case. The value of modification parameter $\kappa$ can be an obstacle for it. We wish to obtain an estimate such as

$$
\int_{\left\{x \in \mathbf{R}^{d}: M_{\kappa} f(x)>\lambda\right\}} w(x) d \mu(x) \leq \frac{C}{\lambda} \int_{\mathbf{R}^{d}}|f(x)| M_{\kappa} w(x) d \mu(x) \quad(\kappa>1) .
$$


But this estimate is false. Indeed, we can show that it is false by constructing a counterexample for which we cannot take $\kappa=3$. We give the counterexample and the proof in Appendix.

2.3. Commutator operators. We present some definitions and known results needed for stating our commutator theorems.

Definition 2.6 ([10], p. 466). A singular integral operator $T$ is a bounded linear operator on $L^{2}(\mu)$ with a kernel function $K$ that satisfies the following three properties :

(1) For some appropriately chosen constant $C>0$, we have

$$
|K(x, y)| \leq \frac{C}{|x-y|^{n}}
$$

where $n$ is a constant in the growth condition (2).

(2) There exist constants $\varepsilon>0$ and $C>0$ such that

$$
|K(x, y)-K(z, y)|+|K(y, x)-K(y, z)| \leq C \frac{|x-z|^{\varepsilon}}{|x-y|^{n+\varepsilon}} \text { if }|x-y|>2|x-z| .
$$

(3) If $f$ is a bounded measurable function with a compact support, then we have

$$
T f(x)=\int_{\mathbf{R}^{d}} K(x, y) f(y) d \mu(y) \text { for all } x \notin \operatorname{supp}(f) .
$$

Definition 2.7 ([5], Definition 3.1). Let $0<\alpha<n$. Then we define a fractional integral operator $I_{\alpha}$ by

$$
I_{\alpha} f(x):=\int_{\mathbf{R}^{d}} \frac{f(y)}{|x-y|^{n-\alpha}} d \mu(y)
$$

where $n$ is a constant in the growth condition (2).

It is well known that $T$ is a bounded operator on $L^{p}(\mu)$ if $1<p<\infty$ (see [10]) and $I_{\alpha}$ is a bounded operator from $L^{p}(\mu)$ to $L^{q}(\mu)$ if $1<p<q \leq \infty$ and $1 / q=1 / p-\alpha / n$ (see [5]). The $L^{p}(\mu)-L^{q}(\mu)$-boundedness of $I_{\alpha}$ was firstly proved in a more general form by $\mathrm{V}$. Kokilashvili in $\mathbf{R}^{d}(1<p<q<\infty)$. In general non-homogeneous spaces the $L^{p}(\mu)-L^{q}(\mu)$-boundedness of $I_{\alpha}$ is proved in a general setting $(1<p<q<\infty)$ in [7], see also the monograph by D. Edmunds, V. Kokilashvili and A. Meskhi [2]. In [13] it is also shown that $T$ is a bounded operator on $\mathcal{M}_{q}^{p}(\mu)$ if $1<q \leq p<\infty$ and $I_{\alpha}$ is a bounded operator from $\mathcal{M}_{q}^{p}(\mu)$ to $\mathcal{M}_{t}^{s}(\mu)$ if

$$
1<q \leq p<\infty, \quad 1<t \leq s<\infty, \quad 1 / s=1 / p-\alpha / n \text { and } t / s=q / p .
$$

Next we formulate the commutator results for these operators. 
Proposition 2.8. Suppose that $a \in R B M O$. Let $1<q \leq p<\infty$ and $T$ be a singular integral operator with associated kernel $K$. Then

$$
[a, T] f(x):=\lim _{\varepsilon \rightarrow 0} \int_{|x-y|>\varepsilon}(a(x)-a(y)) K(x, y) f(y) d \mu(y)
$$

can be extended to a bounded operator on $\mathcal{M}_{q}^{p}(\mu)$.

Proposition 2.9. Suppose that $a \in R B M O$. If the parameters $p, q, s, t, \alpha, n$ satisfy (15) and $1<r<\infty$, then

$$
\left[a, I_{\alpha}\right] f(x):=\lim _{\varepsilon \rightarrow 0} \int_{|x-y|>\varepsilon} \frac{(a(x)-a(y))}{|x-y|^{n-\alpha}} f(y) d \mu(y)
$$

can be extended to a bounded operator from $\mathcal{M}_{q}^{p}(\mu)$ to $\mathcal{M}_{t}^{s}(\mu)$.

In proving the vector-valued estimate, we shall need the ones for $I_{\alpha}$ and a singular integral operator $T$.

Proposition 2.10 ([13]). Suppose that the parameters $p, q, s, t, \alpha, n$ satisfy (15) and that $1<r<\infty$. Let $T$ be a singular integral operator. Then we have

$$
\begin{aligned}
& \left\|I_{\alpha} f_{j}: \mathcal{M}_{t}^{s}\left(l^{r}\right)\right\| \leq C\left\|f_{j}: \mathcal{M}_{q}^{p}\left(l^{r}\right)\right\|, \\
& \left\|T f_{j}: \mathcal{M}_{q}^{p}\left(l^{r}\right)\right\| \leq C\left\|f_{j}: \mathcal{M}_{q}^{p}\left(l^{r}\right)\right\| .
\end{aligned}
$$

\section{Weighted Norm Estimates}

In this section we prove estimates with weights.

3.1. $A_{1}$-properties. In the first subsection we remove the growth condition on $\mu$. We consider the following problem.

Problem 3.1. Given $\kappa>1$, find a condition for locally integrable functions $(u, v)$ such that $M_{\kappa} u(x) \leq C v(x)$.

Set $w=\left(M_{a} g\right)^{\delta}$, where $a>1$ and $\delta$ is a real number slightly less than 1 . If the measure $\mu$ satisfies the doubling condition, then as is well-known, $w \in A_{1}(\mu)$, i.e., $M_{\kappa} w(x) \leq C w(x)$ for $\mu$-a.e. But now we are in the non-doubling situation so that, unfortunately, we have to modify the notion of $A_{1}(\mu)$ weights. Only in this subsection we do not have to impose the condition on $\mu$ : it suffices to assume that $\mu$ is just a Radon measure on $\mathbf{R}^{d}$.

Simple calculation yields the following lemma.

Lemma 3.2. Let $\kappa>b>1$. Let $a>C_{b, \kappa}:=b+\frac{4 b^{2}}{\kappa-b}$ and $Q, R \in \mathcal{Q}(\mu)$. If $R$ satisfies both $Q$ and $\mathbf{R}^{d} \backslash \kappa b^{-1} Q$, then we have $Q \subset a b^{-1} R$.

Proof. By considering each component, we may assume that $d=1$. Since in this lemma we do not have to consider the measure $\mu$, we can normalize $Q$ to obtain $Q=[-1,1]$. In that case we have $\ell(R)>\kappa b^{-1}-1$. Thus if $\left(\kappa b^{-1}-1\right)\left(a b^{-1}-1\right)>4$, that is, $a>C_{b, \kappa}$, then we have $Q \subset a b^{-1} R$.

Using this lemma, we will prove 
Theorem 3.3. Let

$$
\kappa>b>1, \quad a>C_{b, \kappa}, \quad 0<\delta<1 \text { and } 0 \leq \varepsilon<\frac{1}{\delta}-1 .
$$

For a locally integrable function $f$ with $M_{a} f(x)<\infty \mu$-a.e. we have

$$
M_{\kappa}\left\{\left(M_{a} f\right)^{\delta(1+\varepsilon)}\right\}(x)^{\frac{1}{1+\varepsilon}} \leq C_{\delta, a, b, \varepsilon, \kappa} M_{b} f(x)^{\delta} .
$$

Here $C$ is a constant depending on $\delta, a, b, \varepsilon, \kappa$ and not on $f$.

Remark 3.4. Before proving this theorem, we collect some corollaries of this theorem.

(1) Letting $\varepsilon=0$, we obtain $M_{\kappa}\left\{\left(M_{a} f\right)^{\delta}\right\}(x) \leq C_{a, b, \delta, \kappa} M_{b} f(x)^{\delta}$. This is a substitute for $A_{1}(\mu)$ weight in our theory. Since we can take $\kappa^{\prime}$ such that $\kappa>\kappa^{\prime}>1$ and $a>C_{\kappa^{\prime}, b}$, we can apply Proposition 2.5 with $u=\left(M_{a} w\right)^{\delta}$ and $v=\left(M_{b} w\right)^{\delta}$, where $h$ is a $\mu$-locally integrable function. Then we obtain

$$
\int_{\left\{x \in \mathbf{R}^{n}: M_{\kappa} f(x)>\lambda\right\}}\left(M_{a} w\right)^{\delta}(x) d \mu(x) \leq \frac{C}{\lambda} \int_{\mathbf{R}^{n}}|f(x)|\left(M_{b} w\right)^{\delta}(x) d \mu(x) .
$$

(2) Suppose that $Q$ satisfies $\mu(\kappa Q) \leq \kappa^{d+1} \mu(Q)$. Then Theorem 3.3 yields

$$
\begin{aligned}
\left(\frac{1}{\mu(\kappa Q)} \int_{Q} M_{a} f(x)^{\delta(1+\varepsilon)} d \mu(x)\right)^{\frac{1}{1+\varepsilon}} \leq\left(C_{\delta, a, b, \varepsilon} \inf _{Q} M_{b} f(x)^{\delta}\right) \\
\leq \frac{C_{\delta, a, b, \varepsilon}}{\mu(Q)} \int_{Q} M_{b} f(x)^{\delta} d \mu(x) \leq \frac{C_{\delta, a, b, \varepsilon, \kappa}}{\mu(\kappa Q)} \int_{Q} M_{b} f(x)^{\delta} d \mu(x) .
\end{aligned}
$$

Thus

$$
\left(\frac{1}{\mu(\kappa Q)} \int_{Q} M_{a} f(x)^{\delta(1+\varepsilon)} d \mu(x)\right)^{\frac{1}{1+\varepsilon}} \leq \frac{C_{\delta, a, b, \varepsilon, \kappa}}{\mu(\kappa Q)} \int_{Q} M_{b} f(x)^{\delta} d \mu(x) .
$$

This is a substitute for the Reverse Hölder's inequality.

Proof of Theorem 3.3. By putting $\delta^{\prime}=\delta(1+\varepsilon)$ and replacing $\delta^{\prime}$ by $\delta$, we can assume $\varepsilon=0$. Fix a cube $Q \in \mathcal{Q}(\mu)$. We decompose $f$ w.r.t to $\kappa b^{-1} Q$ : $f=f_{1}+f_{2}$, where $f_{1}=f \chi_{\kappa b^{-1} Q}$. Noting that $M_{a}$ is weak- $(1,1)$ bounded (cf. Lemma 2.3), we are in the position to use Kolmogorov's lemma. Thus it follows that

$$
\begin{aligned}
& \frac{1}{\mu(\kappa Q)} \int_{Q}\left(M_{a} f_{1}\right)^{\delta}(y) d \mu(y) \\
& \leq C\left(\frac{1}{\mu(\kappa Q)} \int_{\kappa b^{-1} Q}|f(y)| d \mu(y)\right)^{\delta} \leq C\left(M_{b} f(x)\right)^{\delta} .
\end{aligned}
$$


Since $Q$ is arbitrary as long as $x \in Q$, we have $M_{\kappa}\left\{\left(M_{a} f_{1}\right)^{\delta}\right\}(x) \leq C M_{b} f(x)^{\delta}$. Hence the estimate for $f_{1}$ is valid. Let us turn our attention to the estimate of $f_{2}$. By Lemma 3.2, $R \cap\left(\kappa b^{-1} Q\right)^{c} \neq \varnothing$ implies that $Q \subset a b^{-1} R$. Thus for all $y \in Q$ we have

$$
\begin{aligned}
M_{a} f_{2}(y) & =\sup _{\substack{R \in \mathcal{Q}(\mu) \\
y \in b R}} \frac{1}{\mu(a R)} \int_{R}\left|f_{2}(z)\right| d \mu(z) \leq \sup _{\substack{R \in \mathcal{Q}(\mu) \\
Q \subset a b^{-1} R}} \frac{1}{\mu(a R)} \int_{a b^{-1} R}\left|f_{2}(z)\right| d \mu(z) \\
& \leq \sup _{\substack{S \in \mathcal{Q}(\mu) \\
Q \subset b S}} \frac{1}{\mu(b S)} \int_{S}|f(z)| d \mu(z) \leq M_{b} f(x) .
\end{aligned}
$$

Hence we obtain

$$
\frac{1}{\mu(\kappa Q)} \int_{Q}\left(M_{a} f_{2}\right)^{\delta}(y) d \mu(y) \leq C M_{b} f(x)^{\delta} .
$$

This is what we want for $f_{2}$. Combining (19) and (20), we obtain Theorem 3.3.

We state one more corollary of this theorem.

Corollary 3.5. Suppose that the parameters $a, b, \kappa, \delta$ and function $f$ are the same as in Theorem 3.3. $Q \in \mathcal{Q}(\mu)$ satisfies $\mu(\alpha \kappa Q) \leq K \mu(Q)$ for some $\alpha \geq 1$ and $K>0$. Then for any $\mu$-measurable subset $A \subset \alpha Q$ we have

$$
\int_{A} M_{a} f(x)^{\delta} d \mu(x) \leq C\left(\frac{\mu(A)}{\mu(Q)}\right)^{\frac{\varepsilon}{1+\varepsilon}} \int_{Q} M_{b} f(x)^{\delta} d \mu(x),
$$

where $\varepsilon=(1-\delta) / 2 \delta$.

Proof of Corollary 3.5. Indeed, by Remark 3.4 we have

$$
\begin{aligned}
\int_{A} M_{a} f(x)^{\delta} d \mu(x) & \leq\left(\int_{A} M_{a} f(x)^{\delta(1+\varepsilon)} d \mu(x)\right)^{\frac{1}{1+\varepsilon}} \mu(A)^{\frac{\varepsilon}{1+\varepsilon}} \\
& \leq\left(\int_{\alpha Q} M_{a} f(x)^{\delta(1+\varepsilon)} d \mu(x)\right)^{\frac{1}{1+\varepsilon}} \mu(A)^{\frac{\varepsilon}{1+\varepsilon}} \\
& \leq C\left(\frac{1}{\mu(\alpha \kappa Q)} \int_{\alpha Q} M_{a} f(x)^{\delta(1+\varepsilon)} d \mu(x)\right)^{\frac{1}{1+\varepsilon}} \mu(Q)^{\frac{1}{1+\varepsilon}} \mu(A)^{\frac{\varepsilon}{1+\varepsilon}} \\
& \leq C \inf _{x \in Q} M_{\kappa}\left(M_{a} f^{\delta(1+\varepsilon)}\right)(x)^{\frac{1}{1+\varepsilon}} \mu(Q)^{\frac{1}{1+\varepsilon}} \mu(A)^{\frac{\varepsilon}{1+\varepsilon}} \\
& \leq C\left(\int_{Q} M_{b} f(x)^{\delta} d \mu(x)\right)\left(\frac{\mu(A)}{\mu(Q)}\right)^{\frac{\varepsilon}{1+\varepsilon}} \cdot
\end{aligned}
$$

This is the desired estimate. 
3.2. Good $\lambda$-inequality. In this subsection we apply the results in the preceding subsection with $\kappa=\frac{9}{5}$. In the sequel we assume that again $\mu$ satisfies the growth condition. Let $C_{0}:=C_{\frac{9}{5}, \frac{3}{2}}=\frac{63}{2}$, where $C_{b, \kappa}$ is the constant appearing in Lemma 3.2. To simplify the notation of the weighted measure, we use $w(A)$ to denote $\int_{A} w(x) d \mu(x)$, where $w$ is a positive measurable function and $A$ is $\mu$-measurable.

The main result of this subsection is

Theorem 3.6. Suppose that the parameters $\alpha, n, \lambda, \delta, \varepsilon$ satisfy

$$
0 \leq \alpha<n, \quad \lambda>0, \quad 0<\varepsilon<\frac{1}{\delta}-1 .
$$

Assume that $f \in \mathcal{M}_{q}^{p}(\mu)$ and that an increasing sequence of doubling cube $\left\{Q_{j}\right\}$ satisfy (5). Then there is a constant $C>0$ and $\eta_{0}$ depending only on the parameters such that if $0<\eta<\eta_{0}$ we have

$$
\begin{gathered}
\left(M_{36} w\right)^{\delta}\left(\left\{x \in \mathbf{R}^{d}: M^{\sharp, \alpha} f(x) \leq \eta \lambda, N f(x)>2 \lambda\right\}\right) \\
\leq C_{1} \eta^{\frac{\varepsilon}{1+\varepsilon}}\left(M_{\frac{3}{2}} w\right)^{\delta}\left(\left\{x \in \mathbf{R}^{d}: N f(x)>\lambda\right\}\right) .
\end{gathered}
$$

Proof. The proof will be similar to that of Theorem 6.2 in [16] except that we consider the weighted norm inequality. So we omit some details. We set

$$
E_{\lambda}:=\left\{x \in \mathbf{R}^{d}: M^{\sharp, \alpha} f(x) \leq \eta \lambda, \quad N f(x)>2 \lambda\right\}
$$

and

$$
\Omega_{\lambda}:=\left\{x \in \mathbf{R}^{d}: N f(x)>\lambda\right\} .
$$

It can be assumed that $E_{\lambda} \neq \varnothing$, otherwise there is nothing to prove.

Let $x \in E_{\lambda}$. Then by the definition of $E_{\lambda}$, there exists a doubling cube $R=R_{x} \in \mathcal{Q}(\mu, 2)$ such that $m_{R}(|f|)>\frac{5}{4} \lambda$ and $x \in R_{\lambda}$.

Lemma 3.7. Let $\eta$ be sufficiently small and suppose that $S_{x}$ is a doubling cube containing $2 R_{x}$. Then we have $m_{S_{x}}(|f|) \leq \frac{5}{4} \lambda$.

Proof. Suppose to the contrary. Then we can find a sequence of doubling cubes $R_{k}, k=1,2, \ldots$, such that $2 R_{k} \subset R_{k+1}$ and $m_{R_{k}}(|f|)>\frac{5 \lambda}{4}$ for all $k$. By assumption (5), whether $\mu$ is finite or not, we have $\lim _{k \rightarrow \infty} m_{R_{k}}(f)=0$. Let $k$ be a large integer with $\left|m_{R_{k}}(f)\right|<\frac{\lambda}{4}$. This implies that $m_{R_{k}}\left(\left|f-m_{R_{k}}(f)\right|\right)>\lambda$. Since $M^{\sharp, \alpha} f(x) \geq m_{R_{k}}\left(\left|f-m_{R_{k}}(f)\right|\right)>\lambda$ and $x \in E_{\lambda}$, this is a contradiction if $\eta<1$.

Let us return to the proof of Theorem 3.6. By Lindelöf's covering lemma we can find a countable subset $E_{\lambda, 0}$ such that $E_{\lambda} \subset \bigcup_{x \in E_{\lambda, 0}} R_{x}$. Thus we have

$$
\left(M_{a} w\right)^{\delta}\left(E_{\lambda}\right) \leq\left(M_{a} w\right)^{\delta}\left(\bigcup_{x \in E_{\lambda, 0}} R_{x}\right)
$$


Hence it suffices to estimate $\int_{\cup_{x \in F}} M_{x} w(x)^{\delta} d \mu(x)$ for a finite subset $F$ in $E_{\lambda, 0}$ independent of $F$. Using Lemma 2.2, we can take a subset $F_{0} \subset F$ satisfying

$$
\bigcup_{x \in F} R_{x} \subset \bigcup_{x \in F_{0}} \frac{10}{9} R_{x}, \quad \sum_{x \in F_{0}} \chi_{R_{x}}(x) \leq C \chi_{\Omega_{\lambda}}(x) .
$$

Using an argument similar to that in [16] we obtain

$$
\mu\left(E_{\lambda} \cap \frac{10}{9} R_{x}\right) \leq C \eta \mu\left(\frac{10}{9} R_{x}\right)
$$

for all $x \in E_{\lambda, 0}$, provided that $\eta$ is sufficiently small.

By Corollary 3.5 with $\kappa=\frac{9}{5}, \alpha=\frac{10}{9}$ we have

$$
\left(M_{36} w\right)^{\delta}\left(E_{\lambda} \cap \frac{10}{9} R_{x}\right) \leq C \eta^{\frac{\varepsilon}{1+\varepsilon}}\left(M_{\frac{3}{2}} w\right)^{\delta}\left(R_{x}\right) .
$$

Combining (22) and (23), we have the desired result.

As a corollary, by means of a distribution formula, we obtain our next results, where we replaced $\eta^{\frac{\varepsilon}{1+\varepsilon}}$ by $\eta$ and used $M_{36} f(x) \leq M_{\frac{3}{2}} f(x)$ for all $\mu$-measurable functions $f$.

Corollary 3.8. Let $1<p<\infty$. Under the same assumption as in Theorem 3.6 for small $\eta>0$ we have

$$
\begin{aligned}
& \int_{\mathbf{R}^{d}} N f(x)^{p} M_{36} w(x)^{\delta} d \mu(x) \\
& \quad \leq C_{\eta, \delta} \int_{\mathbf{R}^{d}} M^{\sharp, \alpha} f(x)^{p} M_{\frac{3}{2}} w(x)^{\delta} d \mu(x)+C_{2} \cdot \eta \int_{\mathbf{R}^{d}} N f(x)^{p} M_{\frac{3}{2}} w(x)^{\delta} d \mu(x),
\end{aligned}
$$

where $C_{2}$ is dependent not on $\eta$ but on $\delta$.

\section{Proof of Theorem 1.3}

4.1. A technical lemma. To prove Theorem 1.3 we need the following lemma. In what follows we usually use the letters $u, v, w$ to denote the parameters with $1<u, v, w<\infty$. We do not use the letter $u, v, w$ to denote weight functions.

Lemma 4.1. Suppose that $1<v<\infty, 1<u \leq w, \max \left(\frac{1}{v^{\prime}}, \frac{1}{w^{\prime}}\right)<\delta<1$. Then there exists a constant $C$ independent of $Q$ such that

$$
\begin{gathered}
\mu(100 Q)^{\frac{1}{w}-\frac{1}{u}} \int_{\mathbf{R}^{d}}\left(\sum_{j=1}^{\infty}\left|h_{j}(x)\right| M_{\frac{3}{2}}\left(\left|g_{j}\right|^{\frac{1}{\delta}}\right)(x)^{\delta}\right) d \mu(x) \\
\leq C \sup _{R \in \mathcal{Q}(\mu)} \mu(2 R)^{\frac{1}{w}-\frac{1}{u}}\left\|\chi_{R} h_{j}: L^{u}\left(l^{v}\right)\right\|
\end{gathered}
$$

for all $\mu$-measurable functions $g_{j}, h_{j}(j=1,2, \ldots)$ with $\operatorname{supp}\left(g_{j}\right) \subset Q$ and $\left\|g_{j}: L^{u^{\prime}}\left(l^{v^{\prime}}\right)\right\| \leq 1$. 
Proof. Firstly, we estimate

$$
\mu(100 Q)^{\frac{1}{w}-\frac{1}{u}} \int_{50 Q}\left(\sum_{j=1}^{\infty}\left|h_{j}(x)\right| M_{\frac{3}{2}}\left|g_{j}\right|^{\frac{1}{\delta}}(x)^{\delta}\right) d \mu(x) .
$$

This is easily done by using Hölder's inequality and Lemma 2.1.

$$
\begin{aligned}
& \mu(100 Q)^{\frac{1}{w}-\frac{1}{u}} \int_{50 Q}\left(\sum_{j=1}^{\infty}\left|h_{j}(x)\right| M_{\frac{3}{2}}\left|g_{j}\right|^{\frac{1}{\delta}}(x)^{\delta}\right) d \mu(x) \\
& \leq \mu(100 Q)^{\frac{1}{w}-\frac{1}{u}}\left\|\chi_{50 Q} h_{j}: L^{u}\left(l^{v}\right)\right\| \cdot\left\|\chi_{50 Q} M_{\frac{3}{2}}\left(\left|g_{j}\right|^{\frac{1}{\delta}}\right): L^{\delta u^{\prime}}\left(l^{\delta v^{\prime}}\right)\right\|^{\delta} \\
& \leq C \sup _{R \in \mathcal{Q}(\mu)} \mu(2 R)^{\frac{1}{w}-\frac{1}{u}}\left\|\chi_{R} h_{j}: L^{u}\left(l^{v}\right)\right\| .
\end{aligned}
$$

Thus the estimation of the integral over $50 Q$ is finished.

In what follows we concentrate on the integral over $\mathbf{R}^{d} \backslash 50 Q$ :

$$
\mathrm{I}:=\mu(100 Q)^{\frac{1}{w}-\frac{1}{u}} \int_{\mathbf{R}^{d} \backslash 50 Q}\left(\sum_{j=1}^{\infty}\left|h_{j}(x)\right|\left\{M_{\frac{3}{2}}\left(\left|g_{j}\right|^{\frac{1}{\delta}}\right)(x)\right\}^{\delta}\right) d \mu(x) .
$$

By using Hölder's inequality once more and noticing that $u \leq w$, we have

$$
\mathrm{I} \leq \mu(Q)^{\frac{1}{w}-\frac{1}{u}}\left(\int_{\mathbf{R}^{d} \backslash 50 Q}\left(\sum_{j=1}^{\infty}\left|h_{j}(x)\right|^{v}\right)^{\frac{1}{v}} \cdot\left(\sum_{j=1}^{\infty}\left\{M_{\frac{3}{2}}\left(\left|g_{j}\right|^{\frac{1}{\delta}}\right)(x)\right\}^{\delta v^{\prime}}\right)^{\frac{1}{v^{\prime}}} d \mu(x)\right):=\mathrm{II} \text {. }
$$

By the definition of $M_{\frac{3}{2}}$, we have that $M_{\frac{3}{2}}\left(\left|g_{j}\right|^{\frac{1}{\delta}}\right)(x)$ is less than or equal to

$$
\sup _{\substack{R \in \mathcal{Q}(\mu) \\\{x\} \cup Q \subset R}} \frac{1}{\mu\left(\frac{7}{5} R\right)} \int_{Q}\left|g_{j}(x)\right|^{\frac{1}{\delta}} d \mu(x)=\left(\int_{Q}\left|g_{j}(z)\right|^{\frac{1}{\delta}} d \mu(z)\right) \cdot \sup _{\substack{R \in \mathcal{Q}(\mu) \\\{x\} \cup Q \subset R}} \frac{1}{\mu\left(\frac{7}{5} R\right)}
$$

for all $x \in \mathbf{R}^{d} \backslash 50 Q$. Setting $T_{Q}(x):=\sup _{\substack{R \in \mathcal{Q}(\mu) \\\{x\} \cup Q \subset R}} \frac{\mu(Q)}{\mu\left(\frac{7}{5} R\right)}$ for $x \in \mathbf{R}^{d} \backslash 50 Q$, we find that $M_{\frac{3}{2}}\left(\left|g_{j}\right|^{\frac{1}{\delta}}\right)(x) \leq\left(\frac{1}{\mu(Q)} \int_{Q}\left|g_{j}(z)\right|^{\frac{1}{\delta}} d \mu(z)\right) T_{Q}(x)$ holds. Inserting this into II, we have

$$
\begin{aligned}
\mathrm{II} & \leq \mu(Q)^{\frac{1}{w}-\frac{1}{u}} \int_{\mathbf{R}^{d} \backslash 50 Q}\left(\sum_{j=1}^{\infty}\left|h_{j}(x)\right|^{v}\right)^{\frac{1}{v}}\left(\sum_{j=1}^{\infty}\left(\frac{T_{Q}(x)}{\mu(Q)} \int_{Q}\left|g_{j}(z)\right|^{\frac{1}{\delta}} d \mu(z)\right)^{\delta v^{\prime}}\right)^{\frac{1}{v^{\prime}}} d \mu(x) \\
\leq & \left(\mu(Q)^{\frac{1}{w}-\frac{1}{u}} \int_{\mathbf{R}^{d} \backslash 50 Q}\left(\sum_{j=1}^{\infty}\left|h_{j}(x)\right|^{v}\right)^{\frac{1}{v}} \cdot T_{Q}(x)^{\delta} d \mu(x)\right) \\
& \times\left(\sum_{j=1}^{\infty}\left(\frac{1}{\mu(Q)} \int_{Q}\left|g_{j}(z)\right|^{\frac{1}{\delta}} d \mu(z)\right)^{\delta v^{\prime}}\right)^{\frac{1}{v^{\prime}}} \cdot
\end{aligned}
$$


By Minkowski's inequality and the assumption on $g_{j}$ 's, we have

$$
\begin{aligned}
& \left\{\sum_{j=1}^{\infty}\left(\frac{1}{\mu(Q)} \int_{Q}\left|g_{j}(z)\right|^{\frac{1}{\delta}} d \mu(z)\right)^{\delta v^{\prime}}\right\}^{\frac{1}{v^{\prime}}} \\
& \quad \leq\left(\frac{1}{\mu(Q)} \int_{Q}\left(\sum_{j=1}^{\infty}\left|g_{j}(z)\right|^{v^{\prime}}\right)^{\frac{1}{\delta v^{\prime}}} d \mu(x)\right)^{\delta} \leq \mu(Q)^{-\frac{1}{u^{\prime}}} .
\end{aligned}
$$

Thus it follows that

$$
\mathrm{II} \leq \mu(Q)^{\frac{1}{w}-1} \cdot\left(\int_{\mathbf{R}^{d} \backslash 50 Q}\left(\sum_{j=1}^{\infty}\left|h_{j}(x)\right|^{v}\right)^{\frac{1}{v}} \cdot T_{Q}(x)^{\delta} d \mu(x)\right) .
$$

Denoting the origin by $O$ we obtain by the monotone convergence theorem

$$
\mathrm{II} \leq \lim _{r \rightarrow \infty} \mu(Q)^{\frac{1}{w}-1} \cdot\left(\int_{B(O, r) \backslash 50 Q}\left(\sum_{j=1}^{\infty}\left|h_{j}(x)\right|^{v}\right)^{\frac{1}{v}} \cdot T_{Q}(x)^{\delta} d \mu(x)\right) .
$$

We define $S_{l, r}$ for $r \gg 1$ by $S_{l, r}:=\left\{x \in B(O, r) \backslash 50 Q \mid 2^{-l}<T_{Q}(x) \leq 2^{-l+1}\right\}$, where $l=1,2, \ldots$. Notice that $B(O, r) \backslash 50 Q$ can be partitioned into into a disjoint union of $\left\{S_{l, r}\right\}_{l=1}^{\infty}$, since $T_{Q}(x) \leq 1$ for all $x \in \mathbf{R}^{d} \backslash 50 Q$. Using this partition, we have

$$
\begin{aligned}
\mathrm{II} & \leq C \lim _{r \rightarrow \infty} \mu(Q)^{\frac{1}{w}-1}\left(\int_{B(O, r) \backslash 50 Q}\left(\sum_{j=1}^{\infty}\left|h_{j}(x)\right|^{v}\right)^{\frac{1}{v}} T_{Q}(x)^{\delta} d \mu(x)\right) \\
& \leq C \lim _{r \rightarrow \infty} \sum_{l=1}^{\infty} 2^{-l \delta} \mu(Q)^{\frac{1}{w}-1}\left(\int_{S_{l, r}}\left(\sum_{j=1}^{\infty}\left|h_{j}(x)\right|^{v}\right)^{\frac{1}{v}} d \mu(x)\right) \\
& \leq C \lim _{r \rightarrow \infty} \sum_{l=1}^{\infty} 2^{-l \delta} \mu(Q)^{\frac{1}{w}-1} \mu\left(S_{l, r}\right)^{\frac{1}{u^{\prime}}}\left\|\chi_{S_{l, r}} h_{j}: L^{u}\left(l^{v}\right)\right\| .
\end{aligned}
$$

By virtue of Lemma 2.2 and the definition of $S_{l, r}$, there are cubes $R_{l, r}^{(m)}(m=$ $1, \ldots, N)$ such that $S_{l, r} \subset \bigcup_{m=1}^{N} \frac{6}{5} R_{l, r}^{(m)}$, and that $\mu\left(\frac{7}{5} R_{l, r}^{(m)}\right) \sim 2^{-l} \mu(Q)$, where $N$ is the number independent of $l$ and $r$. Using this covering, we can proceed as follows:

$$
\mathrm{I} \leq C_{N} \lim _{r \rightarrow \infty} \sum_{l=1}^{\infty} 2^{-l\left(\delta+\frac{1}{w}-1\right)} \mu\left(\frac{7}{5} R_{l, r}^{(\alpha)}\right)^{\frac{1}{w}-\frac{1}{u}}\left\|\chi_{\frac{6}{5} R_{l, r}^{(\alpha)}} h_{j}: L^{u}\left(l^{v}\right)\right\| .
$$

By assumption $1 / w^{\prime}<\delta<1$, the series converges, so we have the desired result. 


\subsection{Proof of $1.3(1),(2-a)$.}

Proof of Theorem 1.3 (1), (2-a). Throughout the proof it is assumed that $f_{n} \equiv 0$ for $n$ greater than some $n_{0}$ due to the monotone convergence theorem. In the proof we can always use (5) because we limit ourselves to the proof of Theorem 1.3 (1) and (2-a).

We take an auxiliary $t$ such that $1<t<\min (q, r)$. And we fix the cube $Q$. Then we are to estimate

$$
\mathrm{I}:=\mu(100 Q)^{\frac{1}{p}-\frac{1}{q}}\left(\int_{Q}\left(\sum_{j=1}^{\infty} N f_{j}(x)^{r}\right)^{\frac{q}{r}} d \mu(x)\right)^{\frac{1}{q}}
$$

For this purpose put $u=q / t, v=r / t, w=p / t$, then we have $1<u \leq w<\infty$ and $1<v<\infty$. We write I as follows:

$$
\mathrm{I}=\left\{\mu(100 Q)^{\frac{1}{w}-\frac{1}{u}}\left(\int_{Q}\left(\sum_{j=1}^{\infty}\left(N f_{j}(x)^{t}\right)^{v}\right)^{\frac{u}{v}} d \mu(x)\right)^{\frac{1}{u}}\right\}^{\frac{1}{t}} .
$$

By the duality $L^{v}\left(l^{u}\right)-L^{v^{\prime}}\left(l^{u^{\prime}}\right)$, where $u^{\prime}=u /(u-1)$ and $v^{\prime}=v /(v-1)$, there exists a system of functions $\left\{g_{j}\right\}_{j=1}^{\infty}$ supported by $Q$ such that

$$
\mathrm{I}=\left\{\mu(100 Q)^{\frac{1}{w}-\frac{1}{u}}\left(\int_{Q} \sum_{j=1}^{\infty} N f_{j}(x)^{t} g_{j}(x) d \mu(x)\right)\right\}^{\frac{1}{t}} \text { and }\left\|g_{j}: L^{u^{\prime}}\left(l^{v^{\prime}}\right)\right\|=1 .
$$

Take an auxiliary $\delta$ such that $\max \left(\frac{1}{v^{\prime}}, \frac{1}{w^{\prime}}\right)<\delta<1$. We have from Corollary 3.8 that

$$
\begin{aligned}
\mathrm{I} \leq & C\left\{\mu(100 Q)^{\frac{1}{w}-\frac{1}{u}}\left(\int_{Q} \sum_{j=1}^{\infty}\left(N f_{j}(x)^{t}\left\{M_{36}\left(\left|g_{j}\right|^{\frac{1}{\delta}}\right)(x)\right\}^{\delta}\right) d \mu(x)\right)\right\}^{\frac{1}{t}} \\
\leq & \left\{\mu ( 1 0 0 Q ) ^ { \frac { 1 } { w } - \frac { 1 } { u } } \left(\sum _ { j = 1 } ^ { \infty } \int _ { \mathbf { R } ^ { d } } \left(C_{\eta, \delta} M^{\sharp, \alpha} f_{j}(x)^{t}\right.\right.\right. \\
\left.\left.\left.+C_{2} \eta N f_{j}(x)^{t}\right)\left\{M_{\frac{3}{2}}\left(\left|g_{j}\right|^{\frac{1}{\delta}}\right)(x)\right\}^{\delta} d \mu(x)\right)\right\}^{\frac{1}{t}} & \\
\leq & \left\{C_{\eta, \delta} \mu(100 Q)^{\frac{1}{w}-\frac{1}{u}}\left(\sum_{j=1}^{\infty} \int_{\mathbf{R}^{d}} M^{\sharp, \alpha} f_{j}(x)^{t}\left\{M_{\frac{3}{2}}\left(\left|g_{j}\right|^{\frac{1}{\delta}}\right)(x)\right\}^{\delta} d \mu(x)\right)\right. \\
& \left.+C_{2} \eta \mu(100 Q)^{\frac{1}{w}-\frac{1}{u}}\left(\sum_{j=1}^{\infty} \int_{\mathbf{R}^{d}} N_{f_{j}}(x)^{t}\left\{M_{\frac{3}{2}}\left(\left|g_{j}\right|^{\frac{1}{\delta}}\right)(x)\right\}^{\delta} d \mu(x)\right)\right\} \\
= & \left\{C_{\eta, \delta} \mu(100 Q)^{\frac{1}{w}-\frac{1}{u}} \int_{\mathbf{R}^{d}}^{\infty}\left(\sum_{j=1}^{\frac{1}{t}} M^{\sharp, \alpha} f_{j}(x)^{t}\left\{M_{\frac{3}{2}}\left(\left|g_{j}\right|^{\frac{1}{\delta}}\right)(x)\right\}^{\delta}\right) d \mu(x)\right.
\end{aligned}
$$




$$
\left.+C_{2} \eta \mu(100 Q)^{\frac{1}{w}-\frac{1}{u}} \int_{\mathbf{R}^{d}}\left(\sum_{j=1}^{\infty} N f_{j}(x)^{t}\left\{M_{\frac{3}{2}}\left(\left|g_{j}\right|^{\frac{1}{\delta}}\right)(x)\right\}^{\delta}\right) d \mu(x)\right\}^{\frac{1}{t}} .
$$

We us Lemma 4.1 with $h_{j}(x)=M^{\sharp, \alpha} f_{j}(x)^{t}$ and $h_{j}(x)=N f_{j}(x)^{t}$ respectively to obtain

$$
\mathrm{I} \leq C\left\|M^{\sharp, \alpha} f_{j}(x): \mathcal{M}_{q}^{p}\left(l^{r}\right)\right\|+C_{3} \eta^{\frac{1}{t}}\left\|N f_{j}(x): \mathcal{M}_{q}^{p}\left(l^{r}\right)\right\| .
$$

Since $Q \in \mathcal{Q}(\mu)$ is arbitrary, we have

$$
\left\|N f_{j}(x): \mathcal{M}_{q}^{p}\left(l^{r}\right)\right\| \leq C\left\|M^{\sharp, \alpha} f_{j}(x): \mathcal{M}_{q}^{p}\left(l^{r}\right)\right\|+C_{3} \eta^{\frac{1}{t}}\left\|N f_{j}(x): \mathcal{M}_{q}^{p}\left(l^{r}\right)\right\|
$$

for a sufficiently small $\eta$. Using $\eta$ and as every term of this formula is finite, we have

$$
\left\|N f_{j}(x): \mathcal{M}_{q}^{p}\left(l^{r}\right)\right\| \leq C\left\|M^{\sharp, \alpha} f_{j}(x): \mathcal{M}_{q}^{p}\left(l^{r}\right)\right\| .
$$

This is the desired result.

\section{An Application to Commutators}

In this section we shall extend Propositions 2.8 and 2.9 to $l^{r}$-valued inequalities.

Theorem 5.1. Suppose that $a \in R B M O$. Let $1<q \leq p<\infty, 1<r<\infty$ and $T$ be a singular integral operator with associated kernel $K$. Then

$$
\left\|[a, T] f_{j}: \mathcal{M}_{q}^{p}\left(l^{r}\right)\right\| \leq C\left\|f_{j}: \mathcal{M}_{q}^{p}\left(l^{r}\right)\right\| .
$$

Theorem 5.2. Suppose that $a \in R B M O$. If the parameters $p, q, r, s, t, \alpha, n$ satisfy (15) and $1<r<\infty$, then

$$
\left\|\left[a, I_{\alpha}\right] f_{j}: \mathcal{M}_{t}^{s}\left(l^{r}\right)\right\| \leq C\left\|f_{j}: \mathcal{M}_{q}^{p}\left(l^{r}\right)\right\| .
$$

In the Appendix we consider another type of commutators. The proof of Theorem 5.1 is somehow easier than that of Theorem 5.2. So we prove only Theorem 5.2, while the proof of Theorem 5.1 is omitted.

To prove the theorem we need the following pointwise estimates of commutators.

For details we refer to [16, Section 9] in the case of first assertion and to to $[1$, p. 1293] in the case of second one.

Lemma 5.3. Let $f \in \mathcal{M}_{q}^{p}(\mu)$.

(1) Suppose that $T$ is a singular integral operator and $a$ is an RBMO function. Then

$$
\left(M^{\sharp, 0}[a, T] f\right)(x) \leq C\left\{\left(M_{\left(\frac{4}{3}\right)} f\right)(x)+\left(M_{\left(\frac{4}{3}\right)}(T f)\right)(x)\right\} .
$$

(2) Let $0<\alpha<n$. We have for $\mu$-a.e. $x \in \operatorname{supp}(\mu)$

$$
\left(M^{\sharp, \alpha}\left[a, I_{\alpha}\right] f\right)(x) \leq C\|a\|_{*}\left(M_{\left(\frac{9}{8}\right)}^{\alpha} f(x)+\left(M_{\left(\frac{3}{2}\right)} I_{\alpha} f\right)(x)+I_{\alpha}|f|(x)\right) .
$$


Proof of Theorems 5.1 and 5.2. Since the proofs of these theorems are similar to each other, we prove only Theorem 5.2. Suppose first that $\mu\left(\mathbf{R}^{d}\right)=\infty$. In that case we can use Theorem 1.3, Propositions 2.9, 2.10 and Lemma 5.3 (2). Combining them, we can easily prove the theorem.

Suppose that $\mu\left(\mathbf{R}^{d}\right)<\infty$. Then the treatment of $\left\|M^{\sharp, \alpha}\left[a, I_{\alpha}\right] f_{j}: \mathcal{M}_{t}^{s}\left(l^{r}\right)\right\|$ is the same as in the case $\mu\left(\mathbf{R}^{d}\right)=\infty$.

As for the estimate of the second term

$$
\mathrm{III}:=\left(\sum_{j=1}^{\infty} m_{\mathbf{R}^{d}}\left(\left|\left[a, I_{\alpha}\right] f_{j}\right|\right)^{r}\right)^{\frac{1}{r}}
$$

we have only to show that this is estimated from above by $C\left\|f_{j}: L^{u}\left(l^{r}\right)\right\|$, where $u=\frac{1+\min \left(q, r, \frac{n}{\alpha}\right)}{2}$, since $\left\|f_{j}: L^{u}\left(l^{r}\right)\right\| \leq C\left\|f_{j}: \mathcal{M}_{q}^{p}\left(l^{r}\right)\right\|$. For this purpose define an auxiliary $v$ by $\frac{1}{v}=\frac{1}{u}-\frac{\alpha}{n}$. By using Minkowski's inequality and the boundedness of $\left[a, I_{\alpha}\right]$, III is estimated from above by

$$
\left(\sum_{j=1}^{\infty} m_{\mathbf{R}^{d}}\left(\left|\left[a, I_{\alpha}\right] f_{j}\right|^{v}\right)^{\frac{r}{v}}\right)^{\frac{1}{r}} \leq C\left(\sum_{j=1}^{\infty}\left(m_{\mathbf{R}^{d}}\left(\left|f_{j}\right|^{u}\right)\right)^{\frac{r}{u}}\right)^{\frac{1}{r}} \leq C\left\|f_{j}: L^{u}\left(l^{r}\right)\right\| .
$$

So the proof is finished.

\section{Appendix}

\subsection{A different boundedness of a commutator on the Morrey space.} Finally we consider another commutator with a Lipschitz function and a singular integral operator $T$ or with a Lipschitz function and a fractional integral operator. Shirai [15] considered a commutator with $b \in \Lambda_{\gamma}$ and $T$ and proved the boundedness of $[b, T]$ with the Lebesgue measure. The same proof also holds in our nonhomogeneous space. The proof is similar to the usual case with the aid of Proposition 2.10. For the proof we refer to [15].

Proposition 6.1. Assume that the parameters satisfy

$1<q \leq p<\infty, 1<t \leq s<\infty, \frac{p}{q}=\frac{s}{t}, \frac{1}{s}=\frac{1}{p}-\frac{\alpha+\gamma}{n}, 0<\alpha<n, 0<\gamma \leq 1$.

Suppose that a continuous function $b$ satisfies

$$
|b(x)-b(y)| \leq C|x-y|^{\gamma}
$$

for $C>0$. Then we have

$$
\left\|\left[b, I_{\alpha}\right] f_{j}: \mathcal{M}_{t}^{s}\left(l^{r}\right)\right\| \leq\left\|f_{j}: \mathcal{M}_{q}^{p}\left(l^{r}\right)\right\| .
$$

Proposition 6.2. Assume that the parameters satisfy

$$
1<q \leq p<\infty, \quad 1<t \leq s<\infty, \quad \frac{p}{q}=\frac{s}{t}, \quad \frac{1}{s}=\frac{1}{p}-\frac{\gamma}{n}, \quad 0<\gamma \leq 1 .
$$

Suppose that $b$ is the same function as in Proposition 6.1. Then

$$
\left\|[b, T] f_{j}: \mathcal{M}_{t}^{s}\left(l^{r}\right)\right\| \leq C\left\|f_{j}: \mathcal{M}_{q}^{p}\left(l^{r}\right)\right\| .
$$




\subsection{A note on a weighted norm inequality of Stein-type.}

In considering weighted norm inequalities, it would be the best thing of the following inequality were fulfilled

$$
\int_{\left\{x \in \mathbf{R}^{d}: M_{\kappa} f(x)>\lambda\right\}}|g(x)| d \mu(x) \leq C_{\kappa} \int_{\mathbf{R}^{d}}|f(x)| M_{\kappa} g(x) d \mu(x),
$$

but it will hold only if we make a doubling assumption. Here we disprove (25) with $\kappa=3$ as is announced in Section 2 .

Counterexample of (25). We consider the case $d=2$. We define a measure $\mu$ by a weight function $f$ given below:

$$
f(x)= \begin{cases}1 & (|x| \geq 1) \\ 1 / m ! & \left(2^{-m}<|x|<2^{-m+1} \text { for some } m \in \mathbf{N}\right) . \\ 0 & \text { (otherwise) }\end{cases}
$$

Let $\mu:=f(x) d x$.

We disprove (25) by reductio ad absurdum. Suppose we have inequality (25) with $\kappa=3$. First of all fix an integer $\alpha$. We are going to let $\alpha$ tend to infinity later.

Claim 6.3. Set $R_{m}=\mu\left(B\left(\left(2^{-m}, 0\right), 3 \cdot 2^{-m}\right)\right)^{-1}$. Then we have

$$
B\left(O, 2 \cdot 2^{-m}\right) \subset\left\{(x, y) \in \mathbf{R}^{2}: M_{3} \delta_{O}(x, y)>R_{m}\right\},
$$

where $\delta_{O}$ is a Dirac measure supported on $O=(0,0)$.

Proof. Let $(x, y) \in B\left(O, 2 \cdot 2^{-m}\right)$. By the rotation invariance of the sets $B(O, 2$. $\left.2^{-m}\right)$ and $\left\{(x, y) \in \mathbf{R}^{2}: M_{3} \delta_{O}(x, y)>\lambda\right\}$, we can assume that $0 \leq x<2^{-k+1}$ and $y=0$. Since $O,(x, 0) \in B((x / 2,0),(1+s) x / 2)$ for all $s>0$, we have $M_{3} \delta_{O}(x, 0)>\mu(B((x / 2,0),(1+s) x / 2))^{-1}$. If $s>0$ is sufficiently small, we have $M_{3} \delta_{O}(x, 0)>\mu(B((x / 2,0),(1+s) x / 2))^{-1}>R_{k}$.

It follows from the claim that we have

$$
\int_{B\left(O, 2 \cdot 2^{-m}\right)}|g(x)| d \mu(x) \leq C \mu\left(B\left(\left(2^{-m}, 0\right), 3 \cdot 2^{-m}\right)\right) M_{3} g(O) .
$$

Let $\phi$ be a function such that $\int_{\mathbf{R}^{d}} \phi(x) d x=1$ is supported by a small ball whose center is $O$. For $r \ll 1 / 2$ we take a function $g_{r}$ of the form $g_{r}=$ $\sum_{j=1}^{\alpha} \frac{1}{r^{2}} \phi\left(r^{2} \cdot-x_{j}^{r}\right)$, where $x_{j}^{r}$ satisfies

$$
\lim _{r \rightarrow 0} x_{j}^{r}=\left(2^{-m+1} \cos \frac{2 \pi j}{\alpha}, 2^{-m+1} \sin \frac{2 \pi j}{\alpha}\right)
$$

and

$$
\operatorname{supp}\left(g_{r}\right) \subset B\left(O, 2^{-m+1}\right) \bigcap\left(\bigcup_{j=1}^{\alpha} B\left(\left(2^{-m+1} \cos \frac{2 \pi j}{\alpha}, 2^{-m+1} \sin \frac{2 \pi j}{\alpha}\right), r\right)\right) .
$$


As $r \rightarrow 0$, we have $\alpha \leq C \mu\left(B\left(\left(2^{-m}, 0\right), 3 \cdot 2^{-m}\right)\right) M_{3} \mu_{m, \alpha}(O)$, where denoting by $\delta_{x}$ as the Dirac measure supported by $x$, we set

$$
\mu_{m, p}:=\sum_{j=1}^{\alpha} \delta_{\left(2^{-m+1} \cos \frac{2 \pi j}{\alpha}, 2^{-m+1} \sin \frac{2 \pi j}{\alpha}\right) .}
$$

By the definition of $M_{3} \mu_{m, \alpha}(O)$ we have

$$
=\sup _{\substack{(y, r) \in \mathbf{R}^{n} \times(0, \infty) \\ O \in B(y, r)}} \frac{\sharp\left\{1 \leq j \leq \alpha:\left(2^{-k+1} \cos \left(\frac{2 \pi j}{\alpha}\right), 2^{-k+1} \sin \left(\frac{2 \pi j}{\alpha}\right)\right) \in B(y, r)\right\}}{\mu(B(y, 3 r))} .
$$

For a finite set $J=\left\{j_{1}, \ldots, j_{m}\right\}$ with $1 \leq j_{1}<j_{2}<\ldots<j_{m} \leq \alpha$ we set

$$
\begin{array}{r}
S_{J}:=\inf \left\{\mu(B(y, 3 r)): O,\left(2^{-k+1} \cos \left(\frac{2 \pi j_{1}}{\alpha}\right), 2^{-k+1} \sin \left(\frac{2 \pi j_{1}}{\alpha}\right)\right), \ldots,\right. \\
\left.\left(2^{-k+1} \cos \left(\frac{2 \pi j_{m}}{\alpha}\right), 2^{-k+1} \sin \left(\frac{2 \pi j_{m}}{\alpha}\right)\right) \in B(y, r)\right\} .
\end{array}
$$

Then $M_{3} \mu_{k, \alpha}(O)$ can be written as $M_{3} \mu_{k, p}(O)=\max _{J \subset\{1, \ldots, \alpha\}} \frac{\sharp J}{S_{J}}$.

Fixing $\alpha$, if $\sharp J \geq 2$, we have by geometric observation that $\mu\left(S_{J}\right) \geq \frac{C_{\alpha}}{(m-1) !}$. Notice also that $\mu\left(B\left(\left(2^{-k}, 0\right), 3 \cdot 2^{-k}\right)\right)=O\left(\frac{1}{m !}\right)$. Thus we have

$$
\lim _{k \rightarrow \infty} \frac{\mu\left(B\left(\left(2^{-k}, 0\right), 3 \cdot 2^{-k}\right)\right)}{S_{J}}=0 .
$$

And therefore $S_{\{j\}} \sim \mu\left(B\left(\left(2^{-k}, 0\right), 3 \cdot 2^{-k}\right)\right.$, where $\sim$ does not depend on $\alpha$ and $k$. Thus keeping $\alpha$ fixed, we have

$$
\alpha \leq C \varlimsup_{k \rightarrow \infty} M_{3} \mu_{k, p}(O) \leq C,
$$

where $C$ is independent of $\alpha$. Since $\alpha$ is arbitrary, we have obtained the desired contradiction and (25) is disproved.

\section{ACKNOWLEDGEMENT}

The author is grateful to Dr. Hitoshi Tanaka and Prof. Yasuo Komori for their useful advice and suggestions.

\section{REFERENCES}

1. W. Chen and E. Sawyer, A note on commutators of fractional integrals with $\operatorname{RBMO}(\mu)$ functions. Illinois J. Math. 46(2002), No. 4, 1287-1298.

2. D. Edmunds, V. Kokilashvili, and A. Meskni, Bounded and compact integral operators. Mathematics and its Applications, 543. Kluwer Academic Publishers, Dordrecht, 2002.

3. C. Fefferman and E. Stein, $H^{p}$ spaces of several variables. Acta Math. 129(1972), No. 3-4, 137-193. 
4. N. FujII, A proof of the Fefferman-Stein-Strmberg inequality for the sharp maximal functions. Proc. Amer. Math. Soc. 106(1989), No. 2, 371-377.

5. J. García-Cuerva and A. E. Gatto, Boundedness properties of fractional integral operators associated to non-doubling measures. Studia Math. 162(2004), No. 3, 245-261.

6. V. KokilashviLI, Weighted estimates for classical integral operators. Nonlinear analysis, function spaces and applications, Vol. 4 (Roudnice nad Labem, 1990), 86-103, Teubner-Texte Math., 119, Teubner, Leipzig, 1990.

7. V. Kokilashvili and A. Meskhi, Fractional integrals on measure spaces. Fract. Calc. Appl. Anal. 4(2001), No. 1, 1-24.

8. Y. Komori, Weighted estimates for operators generated by maximal functions on nonhomogeneous spaces. Georgian Math. J. 12(2005), No. 1, 121-130.

9. B. Muckenhoupt, Weighted norm inequalities for the Hardy maximal function. Trans. Amer. Math. Soc. 165(1972), 207-226.

10. F. Nazarov, S. Treil, and A. Volberg, Weak type estimates and Cotlar inequalities for Calderón-Zygmund operators on nonhomogeneous spaces. Internat. Math. Res. Notices 1998, No. 9, 463-487.

11. G. Di Fazio and M. A. Ragusa, Commutators and Morrey spaces. Boll. Un. Mat. Ital. A (7) 5(1991), No. 3, 323-332.

12. Y. SAWANO, Sharp estimates of the modified Hardy-Littlewood maximal operator on the nonhomogeneous space via covering lemmas. Hokkaido Math. J. 34(2005), No. 2, 435-458.

13. Y. Sawano and H. Tanaka, Morrey spaces for non-doubling measures. Acta Math. Sin. Engl. Ser. 21(2005), No. 6, 1535-1544,

14. Y. SAWANo and H. TANAKA, Sharp maximal inequalities and commutators on Morrey spaces with non-doubling measures, Taiwanese J. Math., 2007 (to appear).

15. S. Shirai, Necessary and sufficient conditions for boundedness of commutators of fractional integral operators on classical Morrey spaces, Hokkaido Math. J. (to appear).

16. X. Tolsa, BMO, $H^{1}$, and Calderón-Zygmund operators for non doubling measures. Math. Ann. 319(2001), No. 1, 89-149.

17. H. TRIEBEL, Theory of function spaces. II. Monographs in Mathematics, 84. Birkhäuser Verlag, Basel, 1992.

(Received 27.05.2005)

Author's address:

Graduate School of Mathematical Sciences

The University of Tokyo

3-8-1 Komaba, Meguro-ku

Tokyo 153-8914

Japan

E-mail: yosihiro@ms.u-tokyo.ac.jp 